\title{
A Help for Assisting People Based on a Depth Cameras System Dedicated to Elderly and Dependent People
}

\author{
${ }^{1,2}$ Asma Ben Hadj Mohamed, ${ }^{1}$ Thierry Val, ${ }^{1}$ Laurent Andrieux, and ${ }^{2}$ Abdennaceur kachouri \\ ${ }^{1}$ CNRS-IRIT, Toulouse 2 University, France \\ ${ }^{2}$ Laboratory of Electronic and Technical Information, National School of Engineers of Sfax, Tunisia \\ asma.ben-hadj-mohamed@irit.fr; val@irit.fr; laurent.andrieux@univ-tlse2.fr \\ Abdennaceur.Kachouri@enis.rnu.tn
}

\begin{abstract}
In this paper, we propose a help to a comfort system development based on Kinect sensor to assist people commanding their own house by using only their gestures. The system uses a multi-sensors to detect the person, recognize her gestures and communicate through an IP/KNX gateway to act on actuators related to the home. Thus, a simple gesture is able to turn on/off the lights, to switch on/off the TV, to move up or down the shutters etc. We performed a test bed within the smart home of our University Institute of Technology in Blagnac.
\end{abstract}

Keywords: home automation; Kinect; elderly control; sensor network; smart home; gesture recognition

\section{Introduction}

In recent years, the decline of mortality and fertility rates at the same time has made the life expectancy increased. Therefore, the proportion of the elderly population raised up remarkably all over the world especially in European Union and Canada (see Figure 1). The rate of elderly population is increasing and is estimated to reach almost $30 \%$ of the population in Canada in 2060 [1]. This aging has many impacts on the physical and cognitive health, the economy of the country and the health care spending [2][3][4]. This has engaged the researchers to study the phenomenon and to seek solutions to monitor, control and help to comfort for this category of people.

Home automation is a field of research that aims to keep the elderly or disabled healthy and sound in their own home and facilitate their daily tasks. With the emergence of technology in our daily lives, it's time to share it with these people for their own welfare. However, it's necessary to respond to their needs. The most important is to present a soft, simple, not expensive and especially not intrusive solution.

The classical idea of an accompanying nurse might interfere with patient privacy. However, using a sensor network to collect various data relating the state of the person, or supervising any change in her environment, represents a very advantageous solution for several reasons. On the economic front, monitoring an elderly or disabled person while keeping him in his natural environment would reduce the costs of assistance compared to specialized hospital staff, or to a nurse at home. A significant contribution concerns the psychological and social side of the dependent person, to whom this discreet DOI: $10.14738 /$ jbemi.16.782

Publication Date: $2^{\text {st } J a n u a r y ~} 2015$

URL: http://dx.doi.org/10.14738/jbemi.16.782 
Asma Ben Hadj Mohamed, Thierry Val, Laurent Andrieux, and Abdennaceur kachouri.; A Help for Assisting People Based on a Depth Cameras System Dedicated to Elderly and Dependent People. Journal of Biomedical Engineering and Medical Imaging, Volume 1, No. 6, Dec (2014) , pp 56-66

and imperceptible monitoring provides a sense of security, privacy respect and autonomy [5]. In this paper, we present a part of a generic work whose topic is the monitoring of elderly people living in a smart home. The overall objective is maintaining these persons at home and giving them the feeling of safety and comfort.

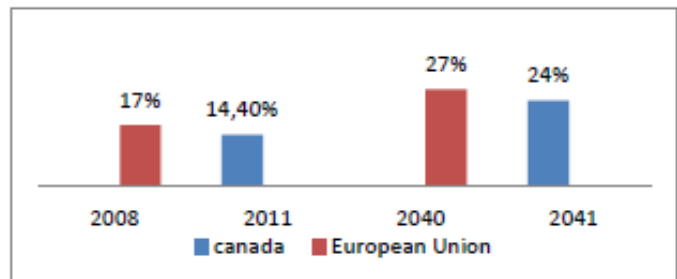

Figure 1: Percentage of people aged more than 65 in EU and Canada, and forecasts by 2040 [1][2]

\section{The Existing Solutions}

The technological revolution in electronics, computer science and wireless sensors networks, has enabled many changes in the medical field, which has greatly evolved over the last years. Indeed, the progress in biomedical instrumentation, implants, prosthetics, biomaterials and medical computing are impressive. Other branches of medicine in continuous progress are telemedicine and health services, such as home tele-monitoring, home automation, health care networks and management of medical tests multi-center. Thus, this positive evolution of medical technologies makes a big business for large companies.

Some solutions such as biometric sensors, who can be integrated on patient's body and control their medical states, are commonly used. In many cases, these devices must be closed or fixed on the body y using belts, watches or patches [6].They can disturb patients and limit their mobility as well as their comfort [7]. Other commercial solutions exist and do not impede the mobility of people and their liberty. They are efficient to track the movements of the person and they can detect the risk of incidents such as a fall. We mention the LysBox device as a good example. It is a small box which can be fixed on the walls in the rooms of the house and monitor the patient [8]. However, it needs a learning period of a few days or weeks to track and learn the person's habits via an internet connection. Some research projects facilitate domestic works by controlling elderly environment through computer systems and touch interfaces employed everywhere in the house. We cite the most remarkable in the field such DOMUS project [9]. It consists of a complete hardware and software system (servers, software application, computer interfaces, Internet, mobile nodes deployed in the patient's environment) to gather information from temperature, pressure, humidity sensors, and communicate instructions/actions to the home devices to be controlled via internal communication network allowing the exchange of messages between the control system and the devices. Thus, it controls automatically the state of the environment. However, the installation of such a system requires a large number of sensors and devices, with a consequent budget. Another interesting research project is called HOMECARE [10] [11]. It provides indoor monitoring for elderly persons with cognitive disease (such as Alzheimer) or loss of independence. It fits in simple devices (patch and shoe sole) to communicate patient status. Clinical studies and tests have shown that the system is accepted by the patients in $80 \%$ of cases, and reliable in case of home treatment (correct fall detection for $98 \%$ of cases). However, this 
system is less trusted when it is applied to a large mass of people (like in hospitals or nursing homes), since the results demonstrate a false fall down average detection every 4 days for one person. Besides, developed devices are attached to the patient's body even if it's in an indiscreet position, which can impede patients.

We can also quote The Guardian Angel project [12]. It aims to produce miniature sensors, integrated on any media type, and capable of self-sustaining energy from the surrounding. The monitoring of various parameters related to health and environment is at the fore front for applications. The project is being developed in partnership with many strong companies and research laboratories [13].

\section{Our Contribution for Help to Comfort}

Starting on already made project ideas, we thought about a comfort support system for this type of person, easy to install and use, cheaper and able to control the home environment easily. People with reduced mobility can thus perform some actions only with gestures, without having to move or make the slightest effort. We also thought to respect the privacy and the feeling of comfort by not attaching sensors to the body. So, our proposition consists in a system development based on depth camera network. Our solution is able to detect patients at home and recognize their gestures in aim to react to their needs (open or close a door, turn on or turn off the lights, etc.). Thus, the general idea is to design a smart environment controlled by people gestures without using remote control, joysticks, push buttons, keyboards or even touch interfaces. The peculiarity of this application also lies in the use of a sensor initially designed for video game.

\subsection{Improve Kinect sensor features}

The choice of Kinect video sensor was made for its features. Initially dedicated to video games systems, it is now used in many applications such as robotic, industry, video games etc[14]. Indeed, it includes a depth camera which is able to monitor people, by respecting theirs identity and privacy, since 3D images hide body details. In addition, the libraries supplied with the software kit of the sensor allow a wide use of depth, color and skeleton data stream. Until today, three versions of the Kinect hardware have been developed: the Kinect for XBOX, the Kinect for Windows and the Kinect for windows 2.0. Tables 1 details characteristics of each version. We used Kinect for XBOX for our application.

Table 1: KINECT sensors characteristics

\begin{tabular}{|c|c|c|c|}
\hline Property & Kinect for XBOX & Kinect for Windows 1 & Kinect for Window 2.0 \\
\hline Field of View & $57^{\circ} \mathrm{H}, 43^{\circ} \mathrm{V}$ & $57^{\circ} \mathrm{H}, 43^{\circ} \mathrm{V}$ & $70^{\circ} \mathrm{H}, 60^{\circ} \mathrm{V}$ \\
\hline Data interface & USB 2.0 & USB 2.0 & USB 3.0 \\
\hline Depth range & $1.8 \mathrm{~m}-4 \mathrm{~m}$ & $0.4 \mathrm{~m}-4.0 \mathrm{~m}$ & $1920 \times 1080 @ 30 \mathrm{mPS}$ \\
\hline Color stream & $640 \times 480 @ 30 \mathrm{FPS}$ & $640 \times 480 @ 30 \mathrm{FPS}$ & $512 \times 424$ \\
\hline Depth stream & $320 \times 240$ & $320 \times 240$ & $512 \times 424$ \\
\hline Infrared stream & None & None & 4 mic-array \\
\hline Audio stream & 4 mic-array & 4 mic-array & No \\
\hline Tits motor & Yes & Yes & Win 8 \\
\hline Skeleton joints & 20 & Win 7, Win 8 & Yes \\
\hline Supported OS & Win 7 & Yes & 6 \\
\hline Near mode & No & 2 & $\$ 399$ \\
\hline Full skeletons trucked & 2 & $\$ 223$ & \\
\hline Price & $\$ 99$ & & \\
\hline
\end{tabular}


Asma Ben Hadj Mohamed, Thierry Val, Laurent Andrieux, and Abdennaceur kachouri.; A Help for Assisting People Based on a Depth Cameras System Dedicated to Elderly and Dependent People. Journal of Biomedical Engineering and Medical Imaging, Volume 1, No. 6, Dec (2014) , pp 56-66

The kinect sensor operates as follows: first, Kinect emits IR lights. Combinated with the "time to flight" values, reflections of the laser beam are computed using a highly parallel System on Chip called "PS1080". The sensor receives a coded infrared light (IR) as input, and produces a colorized picture, an audio stream and a depth image. All of them are synchronized with a VGA format. The most interesting element of this specific sensor is the depth camera that captures the3D scene. In other words, the pixels of the depth image represent the coordinates $(x, y, z)$ of the scene objects. This depth image is insensitive to variations in brightness and contrast levels. Therefore, it is able to work in a dark room thanks to active infrared lights. Thus, it is very useful for monitoring a person in a dark environment.

\subsection{The smart home}

This work focuses on the detection and recognition of gestures using depth sensor which provides depth images. The smart house is located within the University Institute of technology in Blagnac. A 3D view of this house is shown in Figure 2.

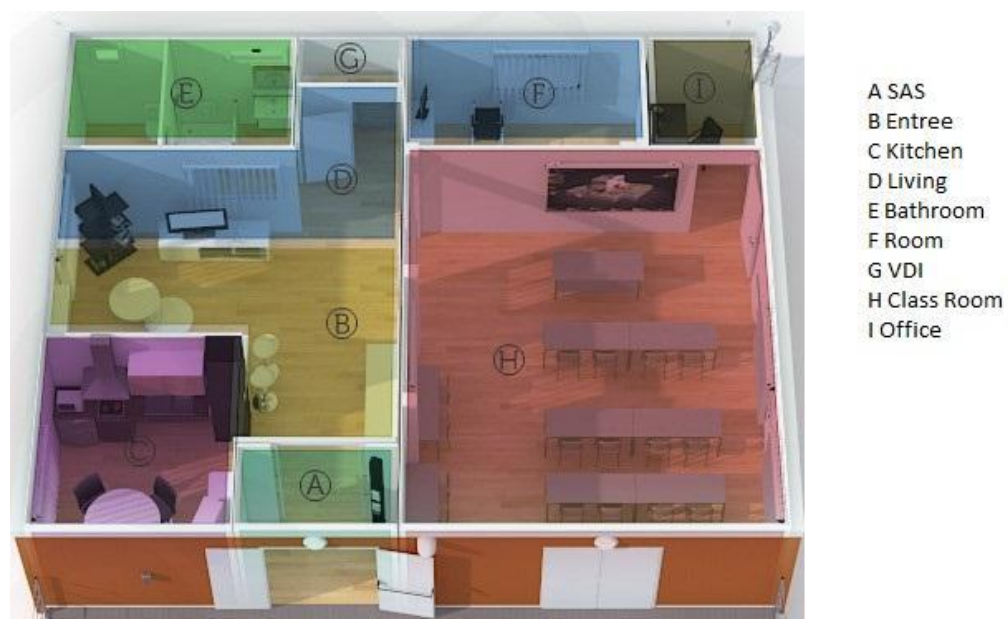

Figure 2: The Smart Home using Kinect sensors C IUT Blagnac [15]

\section{Commanding the Smart Home with Gesture}

\subsection{Gesture recognition using Kinect sensor}

The idea is to implement relatively simple gestures dedicated to these categories of people to make their tasks easier. We made a gesture recognition using hands position. In human body 20 joints are recognized using Kinect sensor [16]. If we compute the coordinates $(x, y, z)$ of each joint in the space we can have an idea about the position of each part of the body referred to the head or spin joint. We have to process the depth and skeleton frame to search the body coordinates of the person, and recognize if hands are below or overhead/spin, or if the patient is showing the right or the left direction.

We have computed the coordinates (in meter) of the right hand in every case. Some of these recognized postures we made are implemented in a database and illustrated in Figure 3 . These gestures will be useful, for example, to turn on/off lights in the living room. We have also implemented a focus gesture based on $\mathrm{Z}$ coordinates in the space. We choose a threshold distance, so if the patient is not moving in the scene, the program is in an idle mode. A push and pull gesture is detected when there is a $20 \mathrm{~cm}$ threshold on the $Z$ axis of each hand. It is illustrated in Figure 4. This gesture will serve to open or close 
the entry door. It is also possible to recognize a gesture as "Hello", or a "skip-like" gesture with hands, in the same way as a person can manipulate a tactile panel. Through these 4 examples, our system is able to interpret many gestures without making the slightest effort.
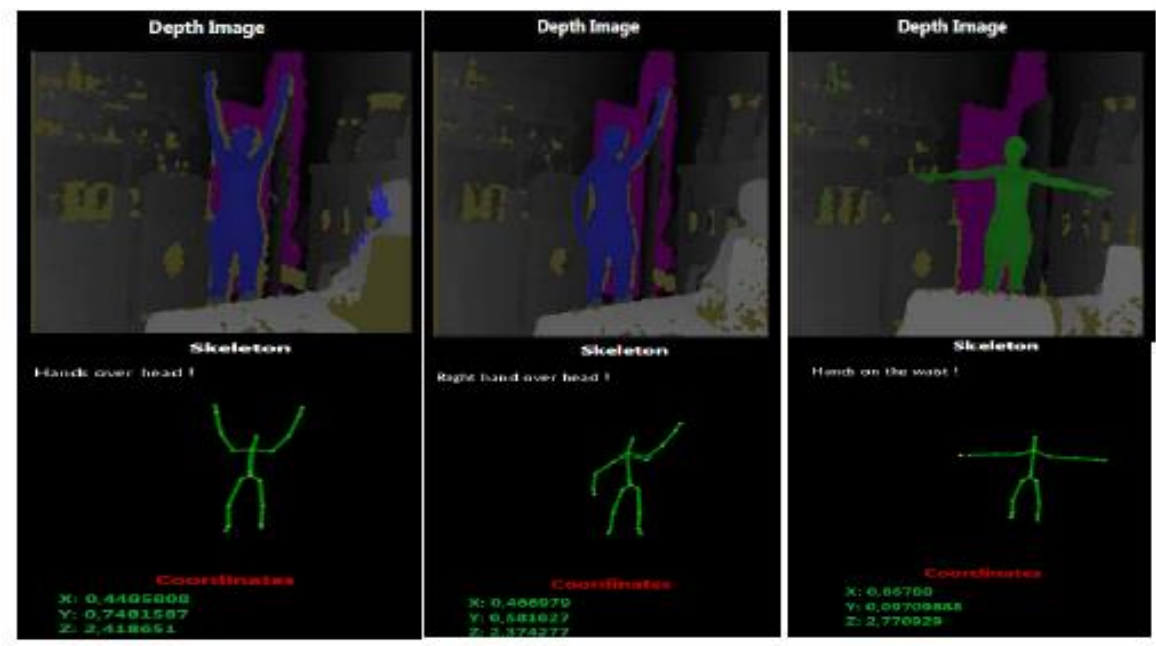

Figure 3: Recognition of 3 different gestures in depth and skeleton frames
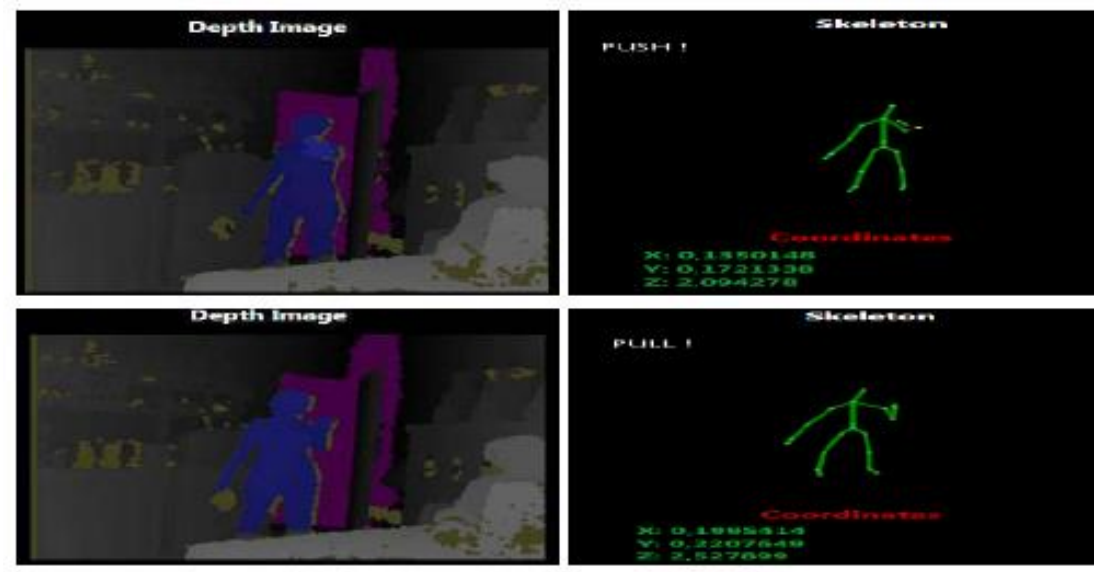

Figure 4: Recognition of push and pull gesture

If we want to recognize a large number of gestures useful in our application, two methods are existing: algorithmic or template based search. The main challenge is to choose the most appropriate method. Neural networks or Hidden Markov Model (HMM) are very sophisticated [9].

However, they require a training (also called learning) time on the wanted form before being saved [10]. This makes these types of algorithms less convenient for prototyping, in which, the user or developer wants to define its own recognizable and recordable form. In addition, this level of sophistication leads to a difficulty in both debugging and programming. However, other algorithms such as the one dollar (\$1) [20] and N dollar (\$N) recognizers [21], can be implemented in any environment, even in a context of fast prototyping [22]. 
Asma Ben Hadj Mohamed, Thierry Val, Laurent Andrieux, and Abdennaceur kachouri.; A Help for Assisting People Based on a Depth Cameras System Dedicated to Elderly and Dependent People. Journal of Biomedical Engineering and Medical Imaging, Volume 1, No. 6, Dec (2014) , pp 56-66

\subsection{Unistroke gesture recognition}

Every gesture is defined by a set of points. These points are compared to another set of points previously stored. From this, it may be deduced that only the comparison between each one of these points is not sufficient to determine the best "candidate point" among sets of points. The templates to recognize gestures are the variations between two gestures made by the same person but at different speeds and / or different figure. They will not generate the same number of points and the comparison is not based only on this single criterion. Furthermore, we have to notice that other problems are occurring: orientation, scale gesture/figure and corners. The \$1 recognizer algorithm [20] is insensitive to all these types of variations. The learning of a gesture is performed only one time, which means it only requires a single pass to create a template unlike Hidden Markov Models (HMM) and neural networks. It should also be noticed that the \$ 1 recognizer considers only "Unistroked" movements, which means a single figure is formed by a continuous gesture, contrary to "Multistroke" movements. The one dollar Unistroke recognizer is defined as four steps. The first three steps are done to create templates for the first time in order to compare them in the immediate future.

- Resampling

- Rotation based on indicative angle

- Scaling and translation

- Get the best score

This score is calculated using the equation:

$$
\text { Score }=1-\frac{d_{i}^{*}}{\frac{1}{2} \sqrt{\left(3 i z e^{2}+\text { size }^{2}\right)}}
$$

Where

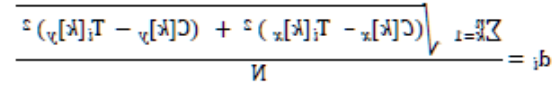

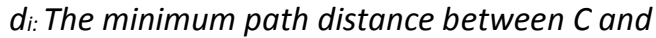

$d_{i:}$ The path distance

C: Candidate points

Ti: The points of each of the templates

Thus, it is possible to integrate another recognizer algorithm such as $\$ 1$ or $\$ N$ into our application. The diagram of the recognition scenario proposed is represented in Figure 5. If an action is detected, we focus on hands position to see if there is a specific gesture that allows to trigger a recognition. The push and pull gesture can be another event allowing to trigger another recognition. We mentioned that the implementation of the $\$ 1$ algorithm will be done in the future work. 


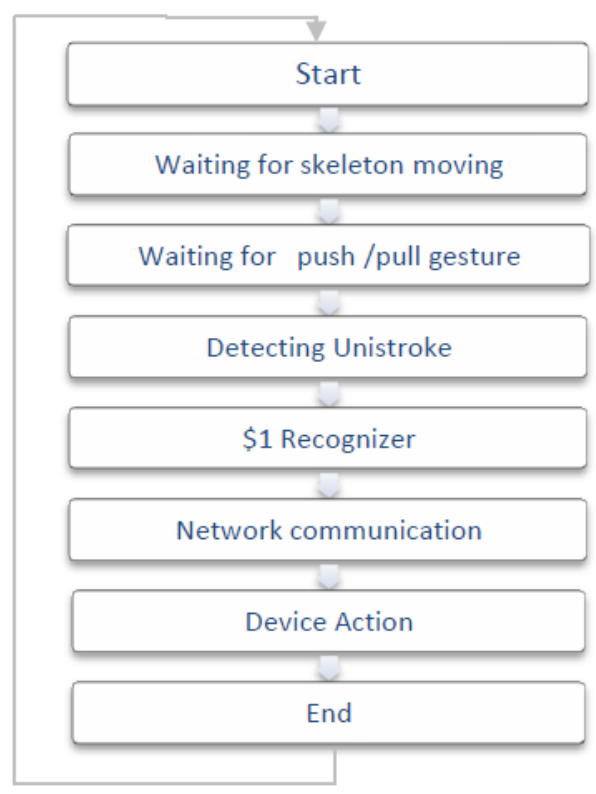

Figure 5: Diagram of gesture recognition using Kinect

At this stage, we used a toolbox included in the Kinect Software Development Kit. This toolbox uses a combination of Golden Section Search and one \$1 method. Therefore, the implementation is able to recognize some other gestures like a circle (see Figure 6).
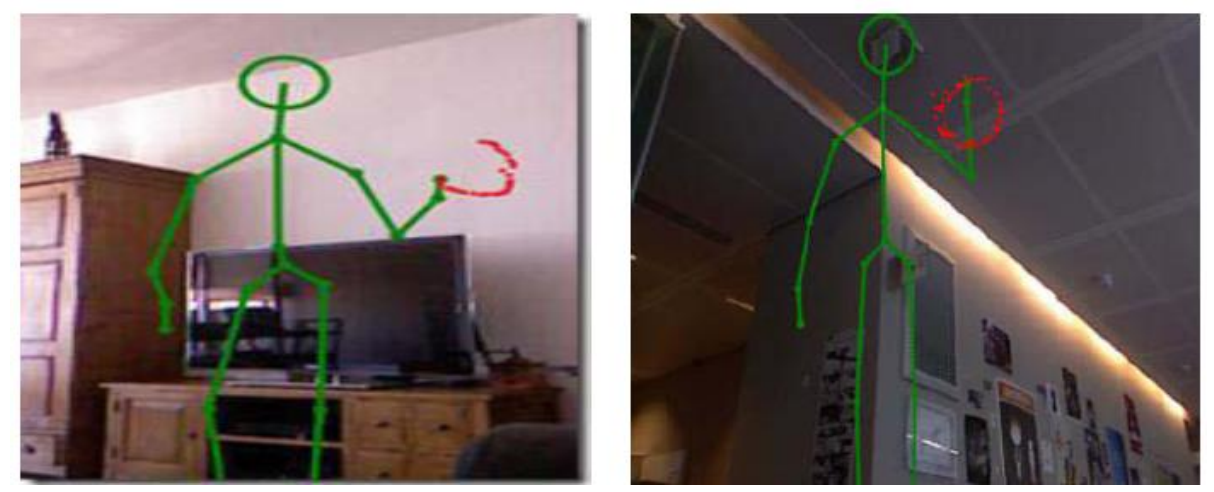

Figure 6: Examples of using Kinect toolbox to recognize a circle gesture [23].

In the next section, we present how we interface the kinect sensors to the actuators, by interconnecting the IP network with KNX bus. We detail how we turn on and off the lights and generate a voice message corresponding to the action.

\section{Network communication}

\subsection{Setting up the Kinect network}

Depending on the size of the house or the hospital, we may need a lot of Kinect sensors that will link to a network (wired Ethernet or wireless WiFi ...). In a parallel work, we have already tested a Kinect wireless sensor network using Mesh WIFI [25]. We have also discussed the use of a WIFI network with mesh topology. Indeed, we test the application of OLSR routing protocol [26] and 802.11s standard [27]. We 
Asma Ben Hadj Mohamed, Thierry Val, Laurent Andrieux, and Abdennaceur kachouri.; A Help for Assisting People Based on a Depth Cameras System Dedicated to Elderly and Dependent People. Journal of Biomedical Engineering and Medical Imaging, Volume 1, No. 6, Dec (2014) , pp 56-66

implemented 802.11s which enables network operation, and we checked its feasibility and validity by getting response time and quality of transmission between wireless nodes.

For each node, we have investigated the response time and the link quality. We also tried to transport Kinect data (depth images) through the network using USB over IP encapsulation [18].

Results show that it is difficult to broadcast data through the mesh network due to the high throughput required in the network which was about $180 \mathrm{MBits} / \mathrm{s}$ [25]. Therefore, we choose to communicate a simple sign. So, when an action is detected, we don't have to communicate all the frames through the network. A specific frame including a text or an alert will be sufficient. We tested our solution by sending an alert signal over the network.

In case of falling down or emergency situation, we send alert frames via the network to see if the patient is safe. Anyway, the transmission of a message within the network seems discreet and easy, and does not represent any kind of the patient spying in their own home. In the next section, we will represent the network architecture between the Kinects connected on IP (Ethernet, WiFi802.11s or a simple WiFi) and the smart home actuators connected on KNX bus.

\subsection{Interconnection IP/KNX}

KNX (KONNEX) or EIBUS (European Installation Bus) is an European standard bus and for building automation [24]. Unlike other automation protocols, it does not work in master/slave mode, but each node is independent of the others. It supports various communication mediums such as Ethernet, radio, and power-line communication. The advantage of using this KNX home automation network is its flexibility when changing the building topology without demolishing walls or installing new circuits and wiring. He brings over some comfort by simplifying everyday life. The system is also cost-effective and provides low-cost installation, globally. The KNX system reacts quickly and deals intelligently in case of emergencies, with the presence or not of the owner. To implement our application, we proposed and realized the network topology shown in Figure 7.

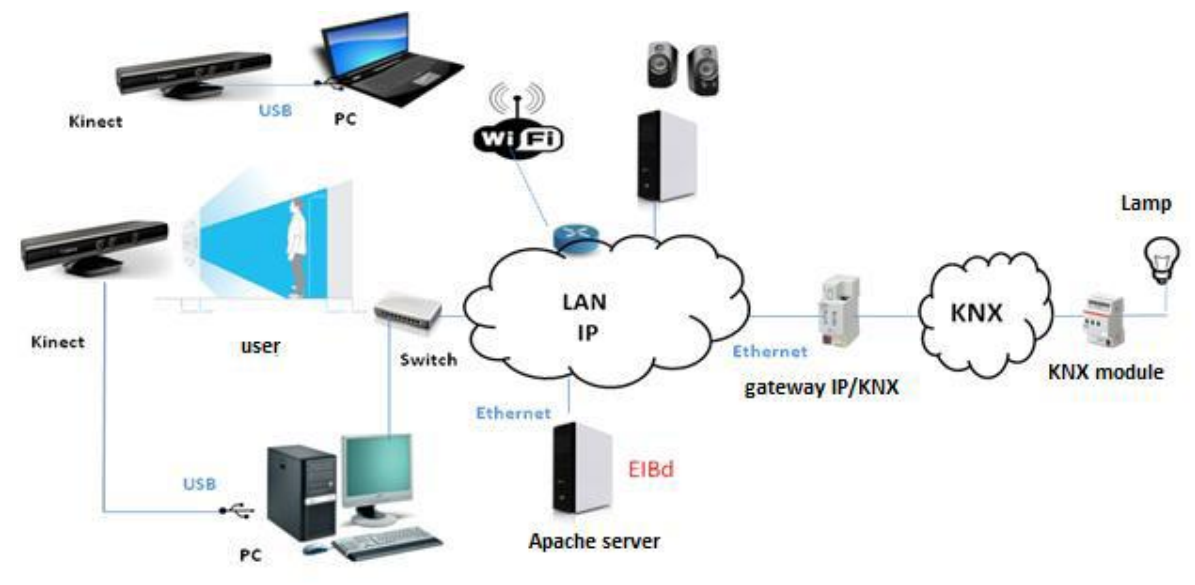

Figure 7: Network Interconnection IP/KNX

We investigated the IP frames transmitted when detection of a gesture occurs in the smart home. The installation of KNX elements is done through the Engineering Tool Software (ETS). The interface between the IP (Ethernet or Wi-Fi) and KNX networks is provided with the IP/KNX hardware gateway. 
The gesture recognition setting ( 1 or 2 hands raised for 3 seconds, for our example) allows to send HTTP requests to a server in order to control the switching of a lamp connected to a KNX module. On the same web server also runs a daemon called EIBd (European Installation Bus Daemon). It is software that interfaces the smart home and the KNX network. The presence of a reactive audio server to these requests also allows controlling a voice synthesizer which confirms the detected gestures and the triggering of corresponded actions.

\subsection{Expanding the monitoring areas}

For a better detection, we can use many Kinect sensors in the same room to expand the monitored area. Therefore, we are interested in linking multiple sensors in order to confirm an alarm detection. Indeed, if we suppose that we have three sensors in the same room, the event will be communicated through the network only if at least two sensors confirm the detection. The data redundancy avoids false detections. In a future work, we will use the IVY bus [29] for data communication between the sensors. It's a light software bus easy to handle in order to connect simple interactive applications written with different languages and running on different machines or platforms [30].

\section{Conclusion}

To continuously control their health, it is necessary to monitor dependent persons especially from afar. Taking care for people with reduced mobility is a part of medicine still in development. An automatic monitoring system seems to be interesting in the medical side. In this paper, we have proposed a solution to assist people using Kinect video sensors. We made a program which detects people gestures, recognize it and communicate it via a network to actuators. We should notice that the Kinect is not intrusive because, it does not film the person details but only her skeleton defined by 20 joints points. In a future work, we will implement an "Unistroke recognizer" [20] to use with Kinect. The main purpose is to implement more gestures to command the smart house using the appropriate algorithm. In prospect, we are currently working on fall detection using a performed 3D bounding box algorithm, to detect elderly falls. The system will be able to react in case of emergency by investigating the patient inactivity and sending alarms to a call center.

\section{REFERENCES}

[1] Emploi et Développement social Canada, http://www4.rhdcc.gc.ca/, 2014

[2] INSEE, Institut Nationale de la Statistiques et des Etudes Economiques, 2013

[3] Munro Dan, The Year In Healthcare Charts, 2012

[4] Dubert H. and Dormont B., Vieillissement de la population et croissance des dépenses de santé Report for the Montparnasse institut, 2012

[5] Wolf C., Maintaining older people at home: Scientific Issues and technologies related tocomputer vision,Etia 2011 
Asma Ben Hadj Mohamed, Thierry Val, Laurent Andrieux, and Abdennaceur kachouri.; A Help for Assisting People Based on a Depth Cameras System Dedicated to Elderly and Dependent People. Journal of Biomedical Engineering and Medical Imaging, Volume 1, No. 6, Dec (2014) , pp 56-66

[6] Conati C., Chabbal R. and Maclaren H., A Study on Using Biometric Sensors for Monitoring User Emotions in Educational Games, Department of Computer Science, University of British Columbia 2366 Main Mall Vancouver, BC, V6T 1Z4, Canada, 2004

[7] BillotA., tutored project, : wireless sensors in biometrics , ISIFC, 2007-2008

[8] http://www.senioractu.com/Loiret-Lysbox-un-boitier-connecte-pour-veiller-sur-les-personnesagees_a17158.html, 2014

[9] Carner P., Project Domus: Designing Effective Smart Home Systems

[10] http://projects.laas.fr/HOMECARE/, 2014-2016

[11] Yohann Charlon, phdthesis, conception d'un dispositif électronique pour l'aide au maintien des personnes âgées , LAASLaboratory, 2014

[12] The Guardian Angel Project, Fet Flagship Pilot, Final report, public version, Federal PolytechniqueSchool of Lausanne, ETH Zurich (Switzerland), April 2012

[13] http://www.industrie-techno.com/guardian-angels-un-projet-europeen-sur-les-capteurs-dufutur.12667, 2012

[14] TölgyessyM., HubinskýP., The Kinect Sensor in Robotics Education, Institute of Control and Industrial Informatics, Faculty of Electrical Engineering and Information Technology, Slovak University of Technology in Bratislava Slovakia, 2010

[15] http://mi.iut-blagnac.fr/

[16] PlyerA., Visual perception and motion estimation, ONERA-DTIM, Paris University 13, Thesis days 2011

[17] KerthoveT., What is the difference between Kinect for Windows \& Kinect for Xbox360, Response of Microsoft to theUK independent review of intellectual property and growth, 4 March 2011.

[18] Anderson D., Bailey C.andSkubicM., Hidden Markov Model symbol recognition for sketch-based interfaces,AAAI Fall Symposium. Menlo Park, CA: AAAI Press, 15-21, (2004)

[19] SezginT.M and DavisR., HMM-based efficient sketch recognition, Proc. IUI '05. New York: ACM Press, 281-283(2005)

[20] WobbrockJ. O., WilsonA .D .andLiY., Gestures without Libraries, Toolkits or Training: A \$1 Recognizer for User Interface Prototypes, University of Washington, UIST'07, October 7-10, 2007, Newport, Rhode Island, USA. ACM 978-1-59593-679-2/07/0010, 2007

[21] http://depts.washington.edu/aimgroup/proj/dollar/

[22] KristenssonP., NicholsonT. and QuigleyA., Continuous Recognition of One-Handed and Two-Handed Gestures using 3D Full-Body Motion Tracking Sensors, School of Computer Science University of StAndrews, St Andrews, United Kingdom, IUI'12, February 14-17, 2012, 
Journal of Biomedical Engineering and Medical Imaging, Volume 1, Issue 6, December, 2014

[23] http://channel9.msdn.com/coding4fun/kinect/ Gestures-and-Tools-for-Kinect-and-matching-Toolkit-too-

[24] http://www.knx.fr

[25] Ben Hadj MohamedA., ValT., AndrieuxL. and KachouriA., Using a Kinect WSN for home monitoring : principle, network and application evaluation, ICWCUCA's, IEEE International Conference on Wireless Communications in Unusual and Confined Areas, Clermont-Ferrand, France, August 2012.

[26] YLi F., VandoniL., Zicca G. and ZanoliS., OLSR Mesh Networks for Broadband Access: Enhancements, Implementation and Deployment, 4th IEEE International Conference on In Circuits and Systems for Communications, ICCSC 200

[27] Joseph CampD. and Edward KnightlyW., The IEEE 802.11s Extended Service Set Mesh Networking Standard, Electrical and computer Engineering, rice University, Houston TX, 2006

[28] HirofuchiT., KawaiE., Fujikawa K. and H. Sunahara, USB/IP - a Peripheral Bus Extension for Device Sharing over IP Netwo, Nara Institute odf Science and Technology, 8916-5 Takayama, Ikoma, 630-0192, Japan, 2005 USENIX Annual Technical Conference

[29] http://www.eei.cena.fr/products/ivy/

[30] Réau. T, 2A2M training report, «Étude et développement d'un dispositif d'interaction multimodale pour I'aide et assistance à domicile », 2014 